\title{
Splenic Torsion in a Twin Premature 4 Month-Old Child Diagnosed by Color Doppler Sonography
}

\author{
Besa Hidri ${ }^{1}$, Dritan Alushani ${ }^{2}$ \\ ${ }^{1}$ Service of Pediatric Imaging, University Hospital Center -Mother Teresa” Tirana, Albania \\ ${ }^{2}$ Pediatric Surgery, University Hospital Center -Mother Teresa” Tirana, Albania.
}

\begin{abstract}
Introduction: Splenic torsion is a vary rare cause of acute abdominal pain in infancy. It is a major, serious and maybe fatal complication of wandering spleen in children. The spleen can "wander" or migrate into various positions within the abdomen or pelvis. The clinical presentation of a wandering spleen is variable. Diagnosis in the emergent setting can be challenging as it is a rare cause of acute abdomen and does not produce any symptoms until splenic torsion has occurred. The perfusion of the spleen is much affected by torsion. Sonoghraphy is an accessible diagnostic method and should be the first choice for identifying wandering spleen especially in children, although CT scan may be needed in certain cases to confirm diagnoses. In wandering spleen, during the sonographic examination, the spleen itself is located in a ectopic position in the abdomen for most cases even though it can also be found in its rightful place, the left upper quadrant. The torsion is the major complication. In case of torsion the spleen can be seen enlarged with parenchimal heterogenicity. Other findings in color Doppler like absence or decreased flow within the spleen, a whorled or tortuous twisted pedicle are important for the right diagnosis of torsion. Splenectomy is the treatment of choice after splenic torsion with infarction. Splenopexy can be performed in patients without splenic infarction. Case report: This is a retrospective case report of a 4 month-old premature-twin child with abdominal pain, distension, diarrhea and a body temperature of $37.5^{\circ}$ Celsius. The sonographic examination revealed an enlarged spleen with a patchy heterogeneous echotexture in the upper left quadrant. Color doppler sonography showed total absence of flow in the splenic vein and artery. The patient underwent surgery. After derotation, viability of spleen appeared compromised. Emergency splenectomy was done. There was no postoperative complication and the patient was followed up in satisfactory condition. Histopathologic evaluation of the spleen revealed nearly complete infarction and congestion of the parenchyma. Conclusion: To conclude, torsion of the spleen is a very rare cause of an acute abdomen in pediatric patients. Diagnosis is challenge because of nonspecific clinical findings. Sonographic examination with color Doppler is the first imaging examination in children. In cases of extremely emergencyit it can help establish the diagnosis. Wondering spleen with torsion, while rare should nevertheless be taken into account when reviewing cases of children with acute abdomen.
\end{abstract}

Keywords: color doppler sonography, torsion, wandering spleen, twin, premature.

\section{Introduction}

Wandering spleen in children is a very rare diagnosis. There are only few articles in literature dealing with this problem. It is more common in females than males in the adult population. The number of children diagnosed with wandering spleen is few accoding to the literature. The occurrence was most common in patients $<1$ year of age, with a 6:1 male predominance in this age category. ${ }^{1}$ The aetiology of wandering spleen may be congenital or acquired. In children, could be congenital due to malformation of the splenic attachments including the gastrosplenic, splenorenal, splenophrenic, splenocolic, splenopancreatic, pancreaticocolic, and phrenocolic ligaments. ${ }^{2}$ During embryology, the splenic ligaments develop from the primitive dorsal mesentery. Developmental embryological anomalies of the spleen's supporting ligaments could be the reason of the wandering spleen.

Splenic torsion is diagnosed in approximately $0.3 \%$ of 1,413 cases of splenectomy in one study of Eraklis, and Filler ${ }^{3}$

Torsion may vary from $1 / 2$ to 6 complete turns around its pedicle. The severity of torsion is depending from the weight of spleen, length of pedicle and degree of ligamentous laxity. There is a wide spectrum of clinical findings, ranging from nonspecific to an acute surgical abdomen, secondary to splenic torsion ${ }^{4}$ and vascular compromise. Physical examination is not diagnostic due to the lack of specific symptoms and signs. The initial method to diagnosing splenic torsion is to image the abdomen using either ultrasonography, especially Doppler sonography or CT imaging. Both methods are sufficient to diagnose splenic torsion and evaluate the vascular supply to the spleen ${ }^{5}$. Duplex ultrasonography and angio spiral CT abdomen are also good diagnostic methods ${ }^{6}$. Magnetic resonance imaging and newer modalities such as liver-spleen scintigraphy, blood pool scintigraphy, and radio-labelled leucocytes have been used olso ${ }^{7,8,9}$

\section{Case report}

A 4- month-old girl was admitted as emergency for ultrasound examination. She had abdominal colics for 3 days. The last day she had developed abdominal distension, diarrhea and a body temperature of $37.5^{\circ}$ celsius. Parents referred that, in the last 2 months, the child had also other abdominal colic episodes. The child was premature, 32 weeks of pregnancy with weight of 1700 gram, born from a twin pregnancy in which the male fetus made exitus in utero with unknown causes.

Physical examination did not reveal any specific finding because of a very distended, and tender abdomen. Laboratory findings: low red blood count $2.500000 / \mathrm{mm}^{3}$. 


\section{International Journal of Science and Research (IJSR) \\ ISSN (Online): 2319-7064}

Index Copernicus Value (2013): 6.14 | Impact Factor (2014): 5.611

Imaging Findings:

X-ray: upright abdominal radiographs revealed dilated loops of bowel without air-fluid levels.

Sonography examination was initially performed with $6 \mathrm{Mhz}$ convex probe and was followed by a focus $9 \mathrm{Mhz}$ linear probe (Fig 1,2,). It revealed an enlarged spleen with a patchy heterogeneous echotexture with multiple altered areas of hypo- and hyperechogenicity (fig. 1,2)

Color Doppler sonography revealed total absence of flow in the splenic parenchyma. Splenic artery and vein were narrowed at the hilum, and no flow was seen (fig.2). The portal vein was founded with normal flow.

The surgeon performed open surgery which revealed: a nearly $720^{\circ}$ torsion around the splenic pedicle, with complete congestion. The splenic ligaments were founded all absent. The spleen was founded free in the left upper quadrant with no attachment to the stomach, retroperitoneum and colon. The abdomen, including the stomach and pancreas appeared normal. An enlarged and totally congestioned infarcted spleen was apparent. In these conditions surgeons could not realized the deterioration of the spleen. Emergency splenectomy was done. Postoperatively the child was vaccinated against pneumococcus and $H$. influenza and treated after with penicillin. There was no postoperative complication and the girl was followed up in satisfactory condition.

Macroscopic pathologic examination of the spleen revealed nearly complete infarction of the parenchyma and marked splenic congestion.(fig 3) Histopathologic examination revealed areas of infarction and congestion of the parenchyma (fig.4,5).

The sonographic, intraoperative, and histopathologic findings were all conclusive with splenic infarction secondary to torsion with no secondary involvement of other organs.

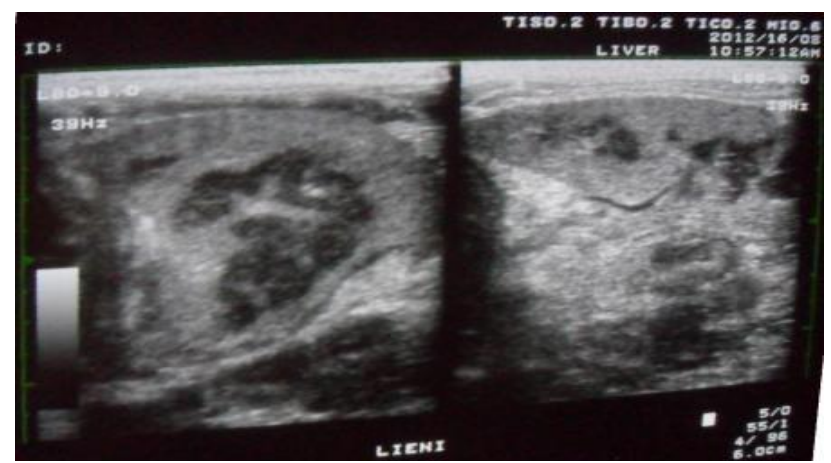

Figure 1: Sonographic examination revealed multiple areas of patchy hypo- and hyperechogenicity of the spleen

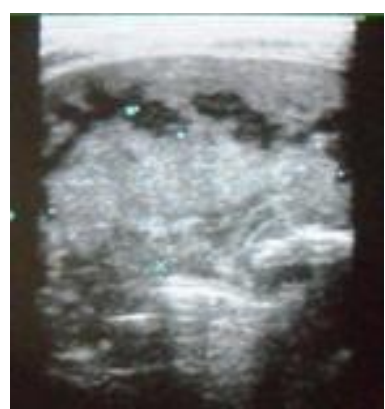

Figure 2: Total absence of flow in the splenic hilum

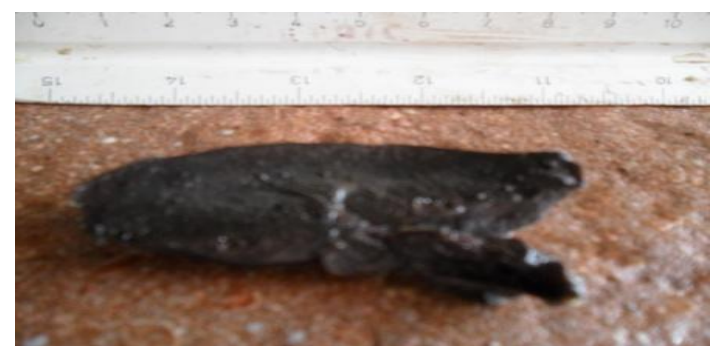

Figure 3: Macroscopic pathological examination of the spleen revealed nearly complete infarction of the parenchyma ( the specimen 1 day after surgery)

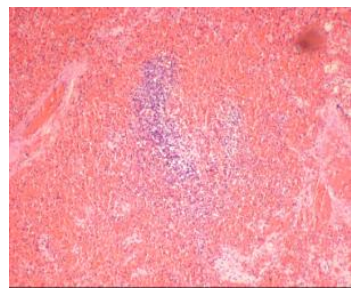

Figure 4: Histopathological examination revealed areas of hemorrhagic necrosis of the spleen

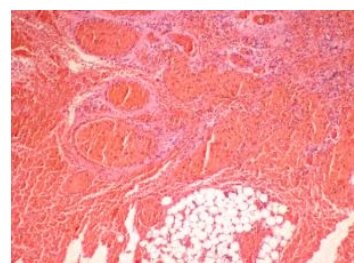

Figure 5: Histopathological examination revealed areas of stasis and intersticial hemorrhage of the spleen

\section{Discussion}

The spleen normally is positioned in the left upper quadrant. It is surrounded by peritoneum and fixed by multiple ligaments. The splenorenal and gastrosplenic ligaments held the spleen in its normal position. These ligaments prevent any displacement of the spleen from its normal position. The splenorenal ligament, which is important contains the splenic artery.

Incomplete development or laxity of the supportive ligaments is the cause of splenic mobility. It can move in an ectopic position and wander everywhere in the abdomen. Absence or laxity of the splenic suspensory ligaments results in increased splenic mobility. In case of torsion the spleen rotate around its long pedicle. ${ }^{10}$ The degree of torsion is founded from $1 / 2$ to 6 complete around its axis. The factors that effect the severity of torsion are the weight of spleen, length of pedicle and degree of ligamentous laxity. In this 


\section{International Journal of Science and Research (IJSR) \\ ISSN (Online): 2319-7064}

Index Copernicus Value (2013): 6.14 | Impact Factor (2014): 5.611

article, we have described a rare case where spleen ligaments were all absent, and no attachement was seen. The spleen was not found in ectopic but in the right position. An enlarged totally infarcted spleen was revealed in open surgery.

Timely identification of the condition and surgical intervention are necessary to prevent complications. The spleen mostly migrates in the left lower quadrant, but other positions can be expected. It is usually intraperitoneal ${ }^{10}$. The causes for the defective suspended ligaments, responsible for wandering spleen are still not very clear.

Wandering spleen is believed to be congenital in children. Splenic torsion should be recognized as a rare cause of an acute abdomen. The condition is very rare. It is reported olso in two sisters, from which the authors suggested a familial association $^{17}$.

In the first year of life there is male predominance (2.5:1), and female predominance thereafter. In our case the girl was born from a twin pregnancy in which the male fetus made exitus in utero with unknown causes. As of the time this article was written, to our knowledge, there is no other reported splenic torsion in a premature twin child in literature. This makes the case reported here unique. As evidenced in our case, color doppler sonography, in certain conditions, can be a precise method for diagnosis particularly in small children.

Sonographic finding for wandering spleen are decisive when the spleen is in an ectopic position. However, if the spleen regains its normal position, the diagnosis may be missed, the condition may recur and result in complications ${ }^{11}$ as in our case report.

Gray scale sonography can reveal an enlarged spleen with a patchy heterogeneous echotexture. The areas of hypo- and hyperechogenicity represent areas of hemorrhage, infarction, and congestion like our case report.

Color doppler sonography shows decreased or absence of perfusion of the spleen. Splenic vein can be seen narrowed and splenic artery can show a high resistive index. Color Doppler evaluation of the blood supply to the spleen ${ }^{12}$ can reveal a whirlpool appearance of splenic vessels ${ }^{13}$. Dynamic sonogram (on the side, standing up) is a special examination that can help define the splenic ptosis. It is also indicated for follow-up and monitoring exams.

CT findings include splenic enlargement from engorgement with little or no contrast enhancement ${ }^{14,15}$. Swischuk et al. describe twisting of the vascular pedicle with a whorled appearance in $\mathrm{CT}^{16}$.

A CT scan is the preferred diagnostic modality for evaluation of the vascular supply to the spleen, especially when there is suspicion of torsion. CT is needed when the spleen cannot be evaluated with sonography because of bowel gas. CT evaluation of the vascular supply to the spleen, can aid in choosing between splenectomy, splenopexy, or nonoperative management ${ }^{17}$.
Magnetic resonance imaging has also been used to make the diagnosis in rare cases ${ }^{18}$.

MRI, if available, is suggested to be the second imaging modality following sonography for children. The splenic vessels at the hilus and surrounding can show a whorl-like appearance on sagittal T1image.

\section{Conclusion}

To conclude, torsion of the spleen is a very rare cause of acute abdomen in pediatric patients. Diagnosis is extremely difficult because of its rarity and nonspecific clinical findings. Imaging examinations, especially color doppler sonography as the imaging examination of choice in children can help establish a diagnosis. A careful and well thought sonographic examination of acute abdomen in children should not discard spleen torsion.

\section{References}

[1] Allen KR, Gay BB, Jr, Skandalakis JE. Wandering spleen: anatomic and radiologic considerations. South Med J 1992;85:976-84

[2] Bekheit M, Katri KM, Ezzat S.Wandering hemi-spleen Laparoscopic management of wandering spleen in a case of polyspleenia.Int J Surg Case report 20123 (5) 151154.

[3] Eraklis AJ, Filler RM. Splenectomy in childhood: a review of 1413 cases. J Pediatr Surg 1972; 7:382-388.

[4] Sodhi KS, Saggar K, Sood BP, Sandhu P. Torsion of a wandering spleen: Acute abdominal presentation. J Emerg.Med.2003;25:133-7.

[5] Fiquet-Francois C, Belouadah $\mathrm{M}$, Ludot $\mathrm{H}$ et al. Wanderin spleen in children : multicenter retrospective study. J Pediatr Surger 2010 45(7):1519-1524

[6] Desai DC, Hebra A, Davidoff AM, Schnaufer L. Wandering spleen: A challenging diagnosis. South Med J 1997;90:439-43.

[7] Arda K, Kizilkanat K, Celik M, Turkalp E. Intermittent torsion of a wandering spleen in a child: The role of MRI in diagnosis. JBR-BTR 2004;87:70-2

[8] Shimizu M, Seto H, Kageyama M, Wu YW, Nagayoshi $\mathrm{T}$, Kamisaki Y, et al. The value of combined $99 \mathrm{mTc}-$ Sn-colloid and $99 \mathrm{~m}$ Tc-RBC scintigraphy in the evaluation of a wandering spleen. Ann Nucl Med 1995;9:145-7.

[9] Posillico LF, Shah AN. A wandering spleen. Detection by in- 111 leukocyte imaging. Clin Nucl Med 1996;21:287-9.

[10] Swischuk LE, Williams JB, John SD. Torsion of wandering spleen: the whorled appearance of the splenic pedicle on CT. Pediatr Radiol 1993; 23:476-477.

[11] Karmazyn B, Steinberg R, Gayer G, Sylvia Grozovski S, Freud E, Kornreich L. Wandering spleen-the challenge of ultrasound diagnosis: Report of 7 cases. J Clin Ultrasound 2005; 33: 433-438

[12] Nemcek AA, Miller FH, Fitzgerald SW. Acute torsion of a wandering spleen: diagnosis by CT and duplex Doppler and color flow sonography. AJR Am J Roentgenol 1991; 157:307-309.

[13] Afshin MOHAMMADI, Mohammad GHASEMI-RAD . Wandering Spleen: Whirlpool Appearance in Color 


\section{International Journal of Science and Research (IJSR) \\ ISSN (Online): 2319-7064}

Index Copernicus Value (2013): 6.14 | Impact Factor (2014): 5.611

Doppler Ultrasonography. A Case Report . MAEDICA a Journal of Clinical Medicine 2015; 10(1): 58-60.

[14] Nemcek AA, Miller FH, Fitzgerald SW. Acute torsion of a wandering spleen: diagnosis by CT and duplex Doppler and color flow sonography. AJR Am J Roentgenol 1991; 157:307-309.

[15] Herman TE, Siegel MJ. CT of acute splenic torsion in children with wandering spleen. AJR Am J Roentgenol 1991; 156:151-153.

[16] Swischuk LE, Williams JB, John SD. Torsion of wandering spleen: the whorled appearance of the splenic pedicle on CT. Pediatr Radiol 1993; 23:476-477.

[17] Ben Ely A, Zissin R, Copel L, Vasserman M, Hertz M, Gottlieb P, et al. The wandering spleen: CT findings and possible pitfalls in diagnosis. Clin Radiol 2006. Nov;61(11):954-958

[18] Arda K, Kizilkanat K, Celik M, Turkalp E. Intermittent torsion of a wandering spleen in a child: the role of MRI in diagnosis. JBR-BTR: organe de la Societe royale belge de radiologie. 2004;87(2):70-72.

\section{Author Profile}

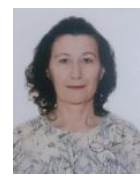

Dr. Besa Hidri has graduated in the Faculty of Medicine with excellent grades. She is professor at Faculty of the Medicine, University of Tirana. Currently works at Pediatric Imaging Service, Imaging Department, University Hospital Center Mother Teresa" Tirana, Albania.

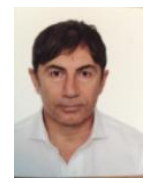

Dr. Dritan Alushani has graduated in the Faculty of Medicine with excellent grades. He is professor at Faculty of the Medicine, University of Tirana. Currently works at Department of Pediatric Surgery, Albania. University Hospital Center -Mother Theresa", Tirana, 